\title{
Precision cosmic ray data from AMS-01
}

\section{Wolfgang Wallraff* $\dagger$}

I. Physikalisches Institut, RWTH-Aachen, Germany

E-mail: (wallraff@physik.rwth-aachen.de)

ABSTRACT: In June 1998 AMS-01, the first magnetic particle spectrometer in space, has been orbiting earth on board of the shuttle "Discovery" for 10 days (last US visit to space station MIR). In an altitude of $380 \mathrm{~km}$ a total of $10^{8}$ triggers have been recorded with all subdetectors operational.

Precision measurements of the two main species of cosmic ray nuclei ( $\mathrm{p}, \mathrm{He}$ ) observed with AMS on "Discovery" while in free flight are reported.

\section{Introduction}

The AMS experiment is designed for an extended space based search for cosmic antimatter and dark matter [1] AMS-01 being the precursor of AMS-02, that will be installed on the International Space Station ISS.

AMS-01 (see Fig.1) is a large acceptance, high resolution, magnetic particle spectrometer $(\mathrm{MDM}=500 \mathrm{GeV}$, $\left.\frac{\Delta \Omega}{4 \pi}=0.35\right)$ in space with some particle identification capabilities $\left(\mathrm{p} / e^{+}\right.$separation by Aerogel threshold Cherenkov counter up to momenta $\mathrm{p}<2.8 \mathrm{GeV}$; $\mathrm{He} / \mathrm{p}$ separation via 10 -fold $\mathrm{dE} / \mathrm{dx}$ measurements in scintillators and Si detectors resulting in a $10^{-7}$ suppression of singly charged particles in the He signal).

After its mission in space AMS-01 has been carefully calibrated in various particle beams at CERN(Geneva)

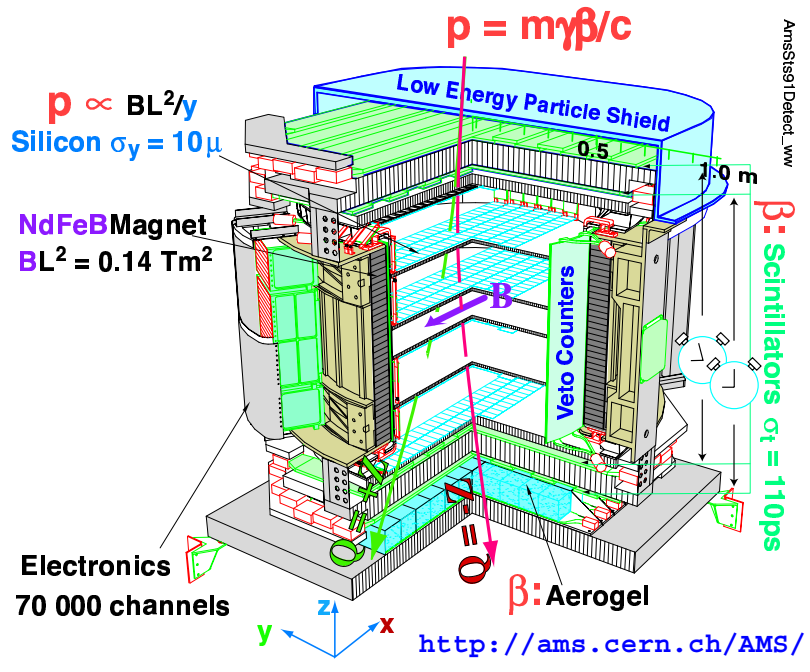

Figure 1: AMS-01 $(\mathrm{p} / \pi, 2<p<14 \mathrm{GeV}$ ) and GSI (Darmstadt) $\left(\mathrm{He} / \mathrm{C}, 1<E_{k i n} / n<6.5 \mathrm{GeV} / \mathrm{n}\right)$.

\footnotetext{
${ }^{*}$ Speaker.

${ }^{\dagger}$ on behalf of the AMS collaboration
} 


\section{Data}

The orbit of the AMS experiment on the spaceflight STS91 servicing MIR, covered all longitudes and geographical (geomagnetic) latitudes up to $52^{\circ}$ $\left(63^{\circ}\right)$.

Almost the full range of geomagnetic cut-offs $\left(15>p_{\text {cut }}>0.5 \mathrm{GeV}\right)$ have been enclosed in the AMS observations. Data were taken continuously (with exceptions at the south atlantic magnetic anomaly) during the free flight of the Discovery orbiter (with respect to zenith at $0^{\circ}\left(20^{\circ}, 45^{\circ}, 180^{\circ}\right)$ for $\left.19(25,20,9) \mathrm{h}\right)$. For control purposes ca. $5 \%$ of the data were transmitted via telemetry. All data were recorded on board the space shuttle on disks and processed after the flight. Technical details of the analysis procedures can be found elsewhere $[2,3]$.

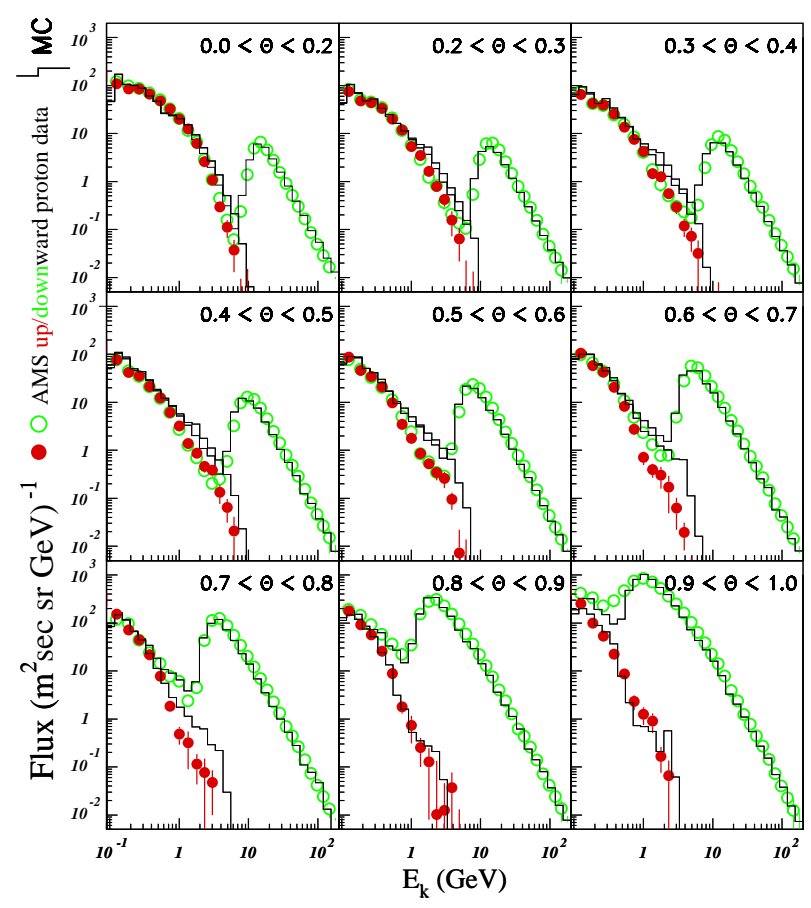

Figure 2: CR protons for geomagnetic slices together with MC simulation [7]

\section{3. cosmic protons}

Protons are the major component of the charged cosmic radiation. At low momenta $(p<5$ $\mathrm{GeV}$ ) the proton flux is known to be strongly influenced by the solar activity, whereas the majority of protons with energies above $15 \mathrm{GeV}$ are of galactic origin [5].

The AMS proton sample is rather pure. Deuterons have been found [6] with the AMS "time of flight" system (about $3 \%$ for momenta up to $1 \mathrm{GeV}$ ) and are rejected from further analysis.

AMS has observed the proton flux with unprecedented statistical accuracy. The accumulated proton flux data are presented in Fig. 2 for slices of geomagnetic latitudes $\Theta_{m}$ up to $1.0 \mathrm{rad}$. The proton flux shows 2 clearly separated components :

- at high momenta protons coming down from zenith ("primary", open symbols)

- at low momenta protons coming from zenith as well as from nadir ("secondary", closed symbols)

Looking at the primary proton flux one can clearly see the expected effect of the geomagnetic cut-off. At the geomagnetic equator $\left(\Theta_{m}=0\right)$ this cut-off has its maximum $(15 \mathrm{GeV})$ and it is softening towards higher geomagnetic latitudes $\left(\propto \cos \left(\Theta_{m}\right)^{4}\right)$. As for the the secondary part the observed values for both up and downgoing fluxes are identical within errors. The secondary flux is apparently trapped in the earth's magnetic field in a 
fashion that is similar to the trapping in the van Allen belt albeit with much larger momenta and magnetic fields. Indeed several groups [7, 8] have presented GEANT based simulations treating the atmosphere like a "magnetized calorimeter" in a beam of the primary cosmic rays. The secondaries from the reactions with the nuclei of the atmosphere are then tracked along the rather precisely known geomagnetic field exhibiting (partial) trapping. The flux values that these simulations predict are in good general agreement with those measured by AMS with an accuracy of about $20 \%$. The biggest discrepancies are in the region of intermediate latitudes $\left(0.2<\Theta_{m}<0.8\right)$. This may be a hint on the fact that the details of the low energy primary flux (solar component) are not modelled with sufficient precision.

The systematic error of the proton flux has three principal contributions:

a) trigger and reconstruction $3.5 \%$

b) MonteCarlo (acceptance and proton selection) $3 \%$

c) spectral unfolding $1 \%$ at $20(100) \mathrm{GeV}[2]$

A total of about $6 \times 10^{6}$ protons have been used for this analysis. In consequence the statistical error is smaller than the systematic one for all AMS proton data the only exception being the upward going flux above $1-2 \mathrm{GeV}$ at $\Theta_{m}>0.3 \mathrm{rad}$ (see Fig. 2).
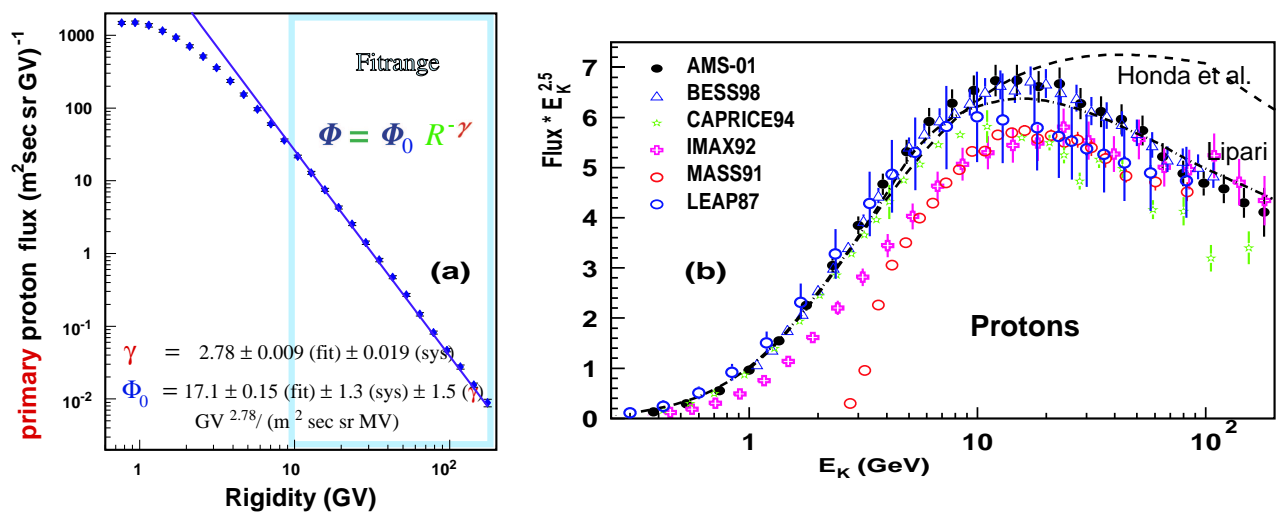

Figure 3: CR protons a) rigidity, b) flux scaled

As shown in Figure 3a the galactic fraction of the proton spectrum follows - as expected - an exponential law in rigidity. All protons observed by AMS with rigidities $\mathrm{R}$ above the geomagnetic cut-off $R_{c}$ have been used to determine the primary proton flux $(\mathrm{R}>$ $\left.R_{c}\left(1.2+2 \sigma\left(R_{c}\right)\right)\right)$ taking the resolution $\sigma\left(R_{c}\right)$ into account. Nevertheless the errors both for the exponent and for the overall flux are dominated by systematics (see insert of Fig. $3 a)$.

Figure $3 \mathrm{~b}$ shows the same flux spectrum scaled by $\left(E_{k i n}\right)^{2.5}$ and as a function of kinetic energy. This is done in order to make fine details more visible and to stretch out the lower part of the spectrum respectively. In a strict sense i.e. at comparable (and low) solar activity AMS [4] and BESS [9] data only can be treated on an equal footing. Furthermore it is clear that older balloon data [5] suffer from normalization problems due to small acceptance, poor resolution and considerable overburden.

These spectra (Fig. 3b) constitute the basic information from which atmospheric neutrino 
flux calculations have to start. As shown by the dashed line, historic models [10] are at variance with the observations especially at high energies and should be replaced by more recent calculations based on the more accurate measurements $[8,11]$.

\section{4. cosmic $\mathrm{He}$}

He nuclei are the second most important component of the cosmic radiation. The galactic $\mathrm{He} / \mathrm{p}$ ratio is limited from below by the primordial ratio, which itself results from the bigbang nucleosynthesis (for further information on the galactic chemical evolution see B.E.J. Pagel [12]). In addition a sizeable contribution is expected to originate from the spallation of heavier cosmic ray nuclei in the interstellar medium.

The He identification for AMS-01 is based on the mass determination via momentum and $\beta$ measurements (Fig. 1) and on the charge determination via energy loss measurements in 4 Scintillator-(ToF) and 6 Si-Tracker-planes (see also AMS publications $[2,13]$ ). The fraction of misidentified He nuclei was less than $10^{-4}$ over the full range of momenta.

During AMS-01 flight a data sample of $2.86 \times 10^{6}$ He nuclei with rigidities in the range of $1<R<140 \mathrm{GV}$ have been gathered and searched through for $\bar{H} e$ nuclei but none have been found. This leads to a $95 \%$ CL upper limit on the $\bar{H} e / H e$ ratio of $1.1 \times 10^{-6}$ [2].

The differential Helium flux, as shown in Fig. 4b, was determined by correcting the observed rates for trigger inefficiencies and for the detector acceptance as a function of particle momentum and direction using a GEANT based Monte Carlo simulation.

The systematic error of the helium flux has three principal contributions:

a) track reconstruction $3 \%$

b) trigger $4 \%$

c) MonteCarlo (He interactions) $2 \%$
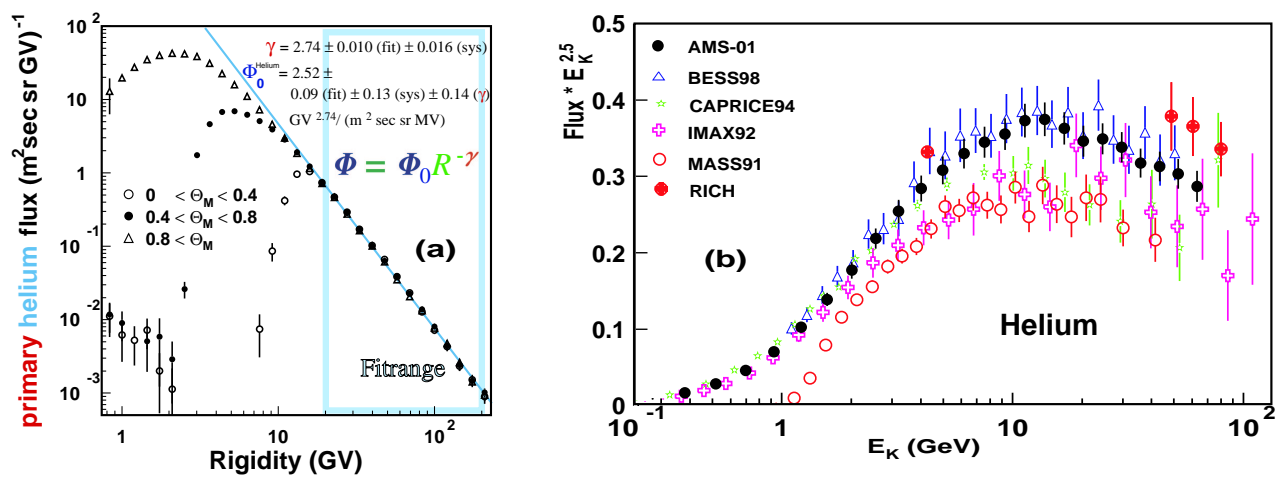

Figure 4: CR Helium a) rigidity, b) flux scaled

Figure $4 \mathrm{a}$ shows the He spectrum and a fit, according to a power law in rigidity $\mathrm{R}$, to the spectrum over the range of $20<R<200 \mathrm{GV}$ together with its fit parameters. Data from the geomagnetic regions with $R_{c u t}>12 \mathrm{GV}$ have been omitted from the fit sample. In the same manner as for the protons (see Fig.2) one can observe a small secondary He flux for $\Theta_{m}<0.8 \mathrm{rad}$ and with rigidities from $0.8 \mathrm{GV}$ (AMS ${ }^{4} \mathrm{He}$ threshold) up to $3 \mathrm{GV}$ 
and an integrated flux of $\approx 10^{-3}\left(m^{2} \cdot s \cdot s r\right)^{-1}$. Detailed analysis of He events with $\beta<$ 0.9 gives evidence that the secondary He spectrum contains a considerable fraction of ${ }^{3} \mathrm{He}$ nuclei [13].

Figure 4b shows the differential He flux spectrum taken with AMS-01 scaled by the He kinetic energy $\left(E_{k i n}^{H e}\right)^{2.5}$. In addition balloon data [5] are plotted. Similarly as for the proton data He fluxes taken with BESS [9] and AMS coincide rather well, whereas earlier ballon data deviate from this precision data by up to $25 \%$.

\section{Summary}

AMS has recorded $10^{8}$ cosmic ray particles during its maiden flight in June 1998. Proton and Helium rigidity spectra have been measured with high precision.

The most important immediate application of the precision cosmic ray flux data can be found in the field of atmospheric neutrino calculations [8, 11]. At the time of this paper definite conclusions cannot be drawn. However, fluxes based on the data prior to AMS and BESS are very likely too shallow in their energy dependence.

From the He data a more stringent limit of $\bar{H} e$ nuclei in the cosmic radiation has been derived [2].

\section{Acknowledgements}

Drs. M. Buénerd, V. Choutko, V. Plyaskin, Prof. Yu. Galaktionov and especially Prof. S.C.C. Ting deserve many thanks for their support of this presentation.

AMS , a multinational effort, has been realized with generous help from DoE, DLR, ASI, CNES as well as Schweizerischer Nationalfonds and especially from NASA at all its levels.

\section{References}

[1] S.P. Ahlen et al. , Nucl. Instrum. Meth. A350 (1994) 351-367;

AMS Coll., W. Wallraff, Lepton and baryon number violation, Trento, 1998, H.V. Klapdor-Kleingrothaus and I.V. Krivosheina eds. 417-444

[2] AMS Coll., J. Alcaraz et al., Phys. Lett. B 461 (1999) 387-396

[3] AMS Coll., J. Alcaraz et al., Phys. Lett. B 472 (2000) 215-226

[4] AMS Coll., J. Alcaraz et al., Phys. Lett. B 490 (2000) 27-35

[5] T. K. Gaisser, astro-ph/0011524, ICRC 2001, Hamburg, Germany, 1643

[6] G. Lamanna, et al. ICRC 2001, Hamburg, Germany, 1614

[7] L. Derome et al., Phys. Lett. B 489 (2000) 1-8,

[8] P. Lipari, astro-ph/0101559

[9] BESS Coll., T. Sanuki et al., Astrophys. J. 545 (2000) 1135

[10] M. Honda, et al., Phys. Rev. D 52 (1995) 4985-5005

[11] P. Lipari, Nucl. Phys. 100 (Proc. Suppl.) (2001) 136-138; V. Plyaskin, Phys. Lett. B 516 (2001) 213-235; T. K. Gaisser, astro-ph/0104327

[12] B.E.J. Pagel, Nucleosynthesis and Chemical Evolution of Galaxies, Cambridge University Press 1997;

M.S. Longair, High Energy Astrophysics, vol. 2, 2nd ed., Cambridge University Press 1994

[13] AMS Coll., J. Alcaraz et al., Phys. Lett. B 494 (2000) 193-202 